\title{
Where do geochemistry teams start and end? Professional and technical support is vital to us all!
}

\author{
By the EAG DEI Committee and the E-DIAL team
}

February 2022

\section{Introduction}

Discoveries in geochemistry reveal important fundamental knowledge of the workings of Earth and solar systems. This understanding can help bring positive change for humanity. We believe that the work that geochemists do is at its best when people of diverse identities and backgrounds develop a sense of belonging to the geochemistry community. But who are our people? Geochemistry teams often list research group members on their institutional and websites and on other public platforms, but does our teamwork extend beyond these bounds? Are we each as individuals, and collectively as a community, seeing, showing appreciation, and including everyone who helps make the geochemical enterprise possible? When passing by, do we stop and properly greet, thank, and respect the people who provide the very foundations that enable outstanding achievements in geochemistry?

People who deliver gas, reagents, and other necessary supplies, as well

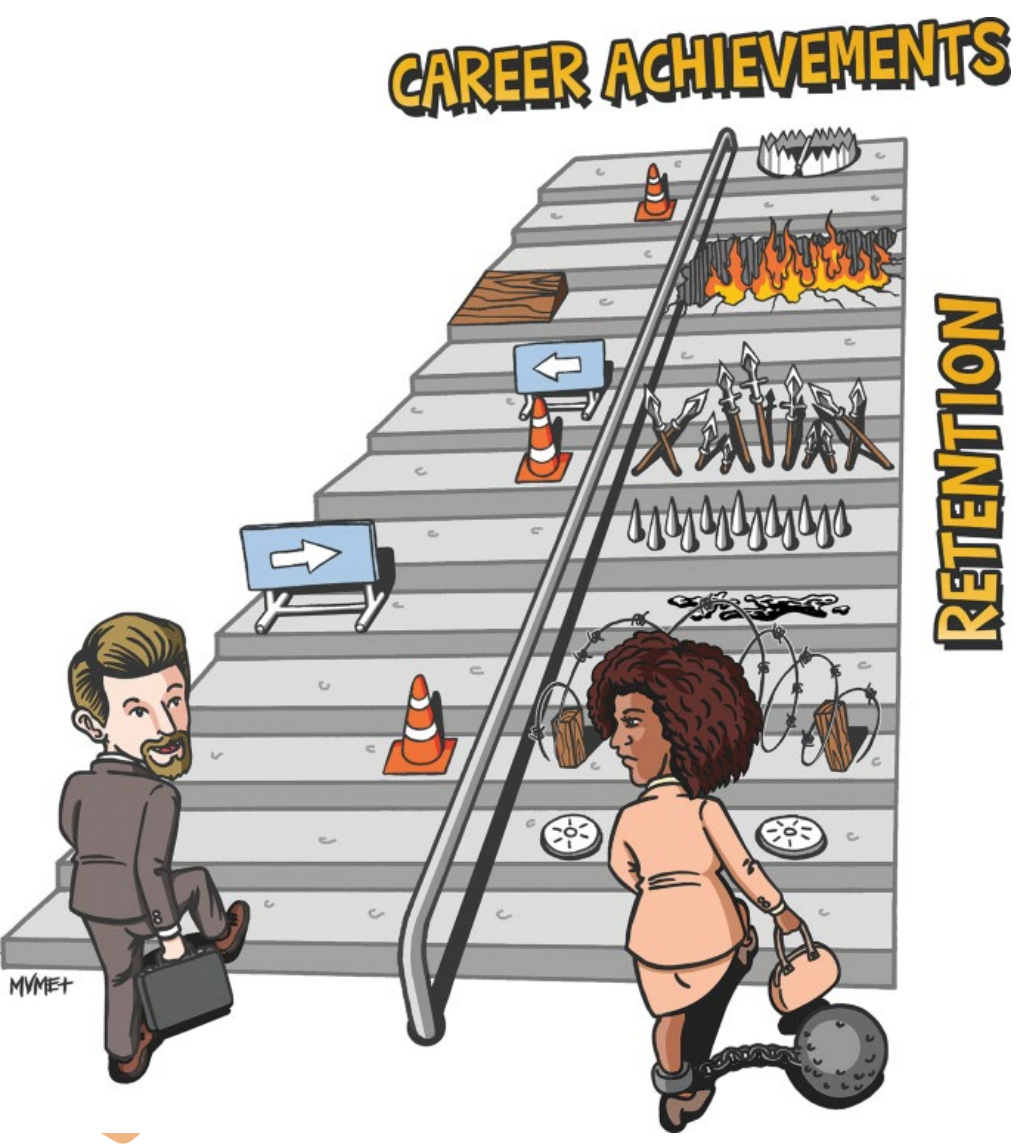

Image Credit: Berhe et al, 2022. Illustration inspired (with permission) by Emanu's Equality hurdles and depicting the hostile obstacle course that women and black, indigenous, and people of colour endure in STEM in many countries. By extension, this framing is relevant to those of other personal characteristics and their intersections such as disability, LGBTQ+, first-language, socioeconomic status, and other factors historically underrepresented or stigmatised in society at large. as those involved in construction, maintenance, cleaning, and security, all help to ensure that our buildings offer safe spaces while basic equipment functions well. Further, woven into the daily duties of geochemists are all forms of accounting, administrative, collections managers, polish section preparators, and technical support personnel, plus senior managers and institutional leaders. These people may never be in our classrooms or trained and approved to access, let alone undertake material processing and analysis in our specialist geochemistry laboratories and with our mass spectrometers or other instrumentation. Yet, their contributions and decision-making are ever present in workplace settings, impacting upon the smooth 
operation of geochemistry as well as the sense of fulfilment and thriving that we seek to draw from our work.

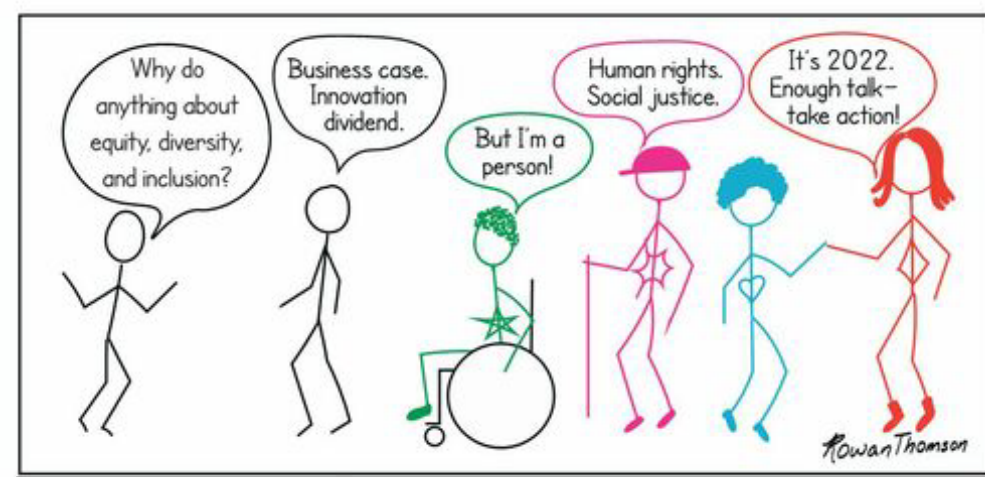

Image Credit: Thomson, 2022. Enough talk - take action.
Many efforts have been made to frame the mole-hills-make-mountains and the obstacle course of challenges in STEM, to progress friendly cultures, improve job security and create scientific ecosystems compatible with good wellbeing, belonging, and positive morale (e.g. EAG DEI Committee, 2021a,b; Riches et al., 2021; Berhe et al., 2022; Limas et al., 2022). However,

all such work can be perceived as hypocritical and ineffectual if we target only trained geochemists or specific groups thereof. We must always give equal attention to those who work with and/or enable the enterprise of geochemistry.

\section{Strong Foundations: Technical and Support Staff}

Key to the success of many geochemists with varied and sometimes highly-specialised needs are high degrees of professionalism, skill, and inclusive conduct among technical and other support staff. These colleagues can be important in being part of collective efforts to identify and tackle challenges to a diverse, inclusive, and equitable geochemistry workforce. During the COVID-19 pandemic, many of these staff have had added duties, and limitations on building access and co-working necessitated changes to work patterns. These adjustments have meant that flexible and home working has been (and in some cases remains) available to

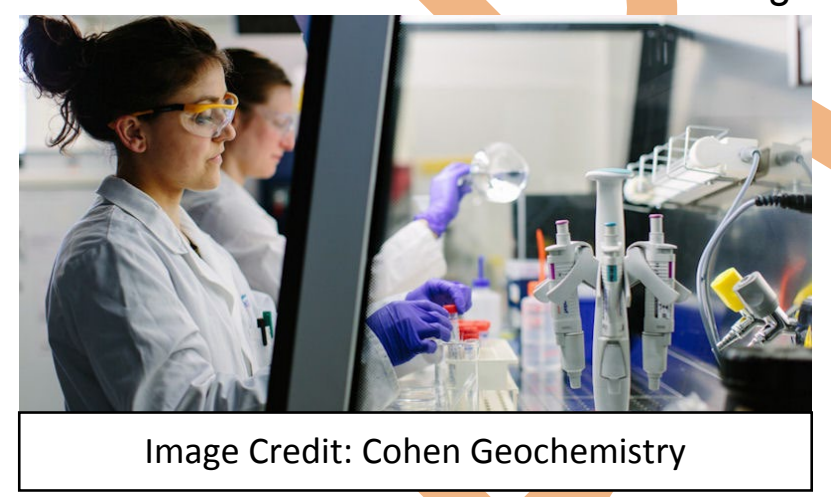

a wider range of roles than was previously possible or acceptable. Because of pandemic circumstances, some technical staff have far exceeded the usual and reasonable expectations in producing geochemical data that are vital to research and for the careers of various team members who were unable to directly access laboratories.

Are good working conditions, intentional support of good mental health and wellbeing, a sense of being valued by team members, and job security necessary to attract and develop the excellence among these staff? In consequence, do these support and technical specialists play critical roles for early career scientists, while further empowering other colleagues? Do we each give proper credit among our publications - through acknowledgements, 'contributorship', or co-authorship - to recognise the input of these colleagues? In so doing, are we recognising the important roles that they play in support of FAIR and open data? Are there competitive advantages for groups in geochemistry and wider STEM that have security and continuity in their support staff (e.g. curators, technical and professional services personnel), research team secretaries, and others? Where these colleagues enjoy stability and working conditions positive for morale, do learning environments, student experiences, and research benefit? 
Despite geochemists relying on them, how often are technical and support personnel featured first or are the focus of our conversations and reports on diversity, equity, and inclusion and (revolutionary) system reforms? For example, though international geochemistry award systems are currently subject to scrutiny and reforms are progressing (e.g. Pourret et al., 2021; Geochem. Soc., 2021; Vance et al., 2021), these awards do not explicitly include technical and/or support personnel as team members or as individuals. The persistence of these stark omissions over many decades testifies to the glacial rate of culture change advantageous to inclusivity; the community must insist that we address this failing with urgency. Yet, even though awards are important in providing role models and reflecting our values, they are a small part of the overall geochemistry ecosystem. It is the day-to-day conditions, practices, available professional development / promotion pathways, and attitudes among geochemists that we must reflect on and do everything possible to consider and lift with equal attention. Only in doing so will we make a true and lasting positive difference for geochemists.

\section{The Importance of Kindness}

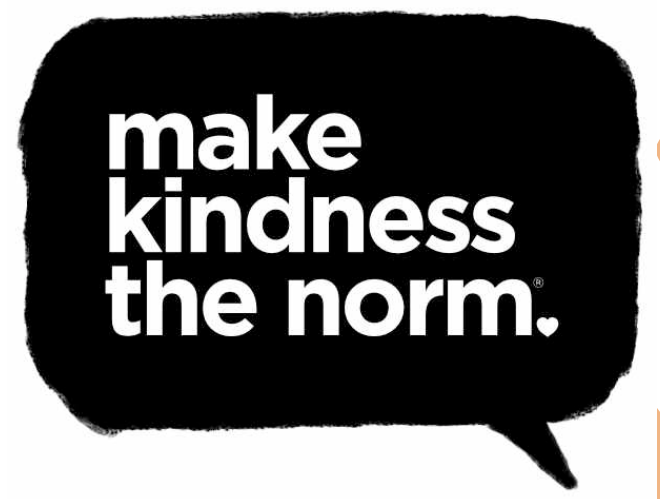

Creating a diverse and inclusive geochemistry community across the globe and among international societies is long overdue. It is the just and right thing to do so that all people can thrive. It is also necessary to ensure that varied ways of thinking are present to achieve our full potential. Underpinning all our efforts in this respect are the values and behaviours of respect toward others and kindness. Often STEM is described as highly competitive, and while there may be truth in this for some performative measures, we must think deeply about how we define success individually and collectively. We should recognise that our best work results from excellent teams and cooperative international working.

The geochemistry community needs people who challenge the glacial pace of reform in how our discipline functions, while reminding us of the unconscious biases that we each possess. How often do we make time for deep self-reflection on every aspect of our conduct, decision making, and professional lives? It is the 2020s and the societies and communities we live in are ever more diverse. So, why have we seen only incremental change in the diversity of STEM communities, particularly over the last half century when global migration has made societies more diverse? Knowing this, why is training in these areas not always taken up at $100 \%$ and often not provided on a mandatory basis? Should we be much more vehement and radical in our approaches?

Institutions and nations can be at different stages of evolution with respect to diversity and inclusion, and varying progress can be attributed to a wide variety of reasons. Institutional and broader diversity and inclusion initiatives have, in some cases, been shown to be flawed. For example, we see change beneficial to white cis heterosexual fully-able (married) women, but not with equal improvements among racial and other minority groups (e.g. Bernard and Cooperdock, 2018; Xiao et al., 2020; Henderson and Bhopal, 2021). Or worse, well-intended 
initiatives sometimes perpetuate the normative practices of majority groups, business case thinking, and neoliberalism (Tzanakou and Pearce, 2019; Bhopal and Pitkin, 2020; Haacker et al., 2022). Such problematic schemes may even set targets that become drivers with the potential for superficial, tokenistic, disingenuous approaches and so-called 'gaming' across staff groups if handled as 'tick box' exercises. In such cases, the very principles of the intended work are undermined and even the ethos of collective endeavour and the power of teamwork may be threatened if hostile workplace atmospheres arise.

We must always acknowledge our mistakes and learn from them. Have such errors been made because we are not yet working extensively and often enough with experts in social sciences, the humanities, and psychology who are best qualified

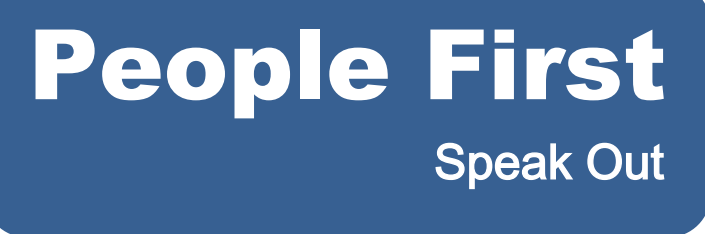
and able to help us improve geochemistry and the wider sciences? Equally, it is commonly stated that approaches and monitoring of matters of diversity and inclusion - where intersectionality is always important - must be made possible with more granular detail, and targeted funding is needed to achieve this. Plus, it is imperative that both positive and negative lived experiences are captured and considered. Awareness of varied experiences ensures that the geochemistry and STEM community, as well as individuals with responsibility to others, are connected to and accountable for the realities of all people. Clearly, we must balance the

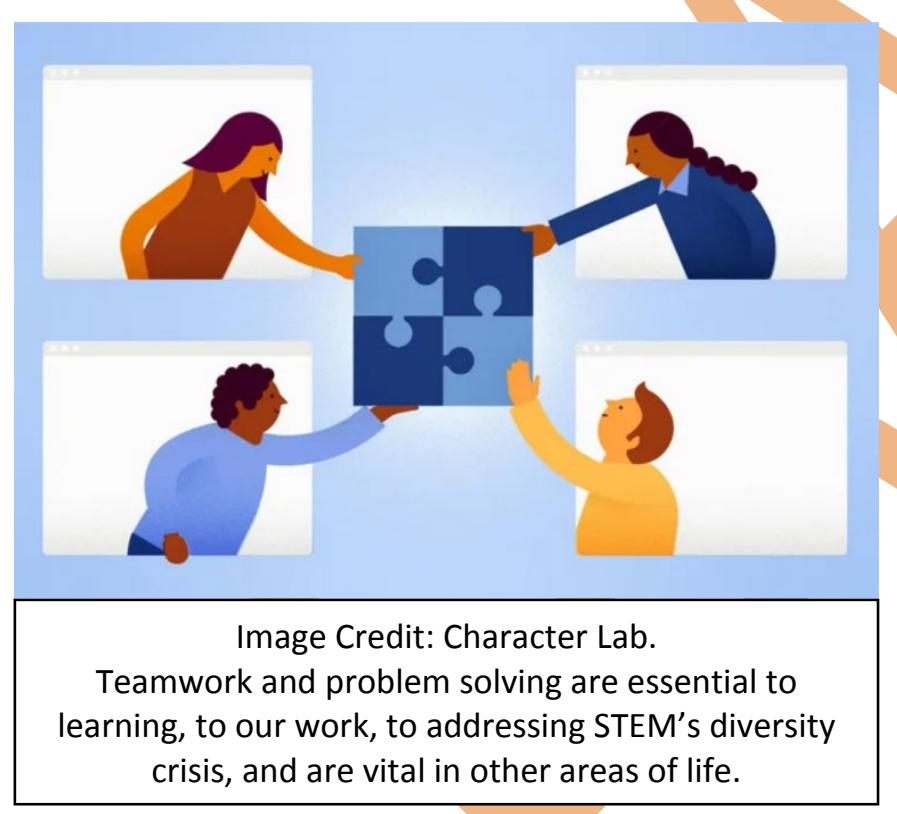
urgent need for change with doing things right. Are we all fairly sharing in the work involved, and are these contributions recognised as part of our professional duties?

Only by working together as a proactive and united force that learns from and listens to all of its people can we make progress together. We hope that the Global Geochemistry Community Survey (https://eag-gs.limesurvey.net/182975), which gathers community data, plays an essential role alongside our community's day-to-day kindness and cooperative conduct. It is imperative to geochemistry's future success that team culture is valued and recognised, and that technical and support staff are priorities in our work to advance inclusion. In drawing strength together, we will drive unstoppable change for better lives now and improved experiences and prospects for geochemists of all generations to come.

\section{Acknowledgements}

Readers of this blog may be interested in this recorded webinar concerning kindness that is available from the Royal Society of Chemistry. 
This contribution was authored by members of the European Association of Geochemistry's Diversity, Equity, and Inclusion Committee in collaboration with the UK's funded E-DIAL team. E-DIAL is a project titled 'Evaluation Diversity and Inclusion within the geochemistry Academic Ladder', supported by the UK's Natural Environment Research Council under grant number 2021EDIE032Anand. This work has been influenced by conversations with the community and among the authors; it is an endeavour that has been fairly shared.

Listed in alphabetical order of last name, contributing authors are:

Pallavi Anand, Francis Appiah, Sandra Arndt, Shonil Bhagwat, Pieter Bots, Ernest Chi Fru, Jessica Gagnon, Jabrane Labidi, Anya Lawrence, Zimin Li, Susan Little, Johanna Marin Carbonne, Elena Maters, Bryne Ngwenya, Olivier Pourret, Amy Riches, and Elias Samankassou.

We are delighted to be poised to announce that new members that have recently joined the European Association of Geochemistry's Diversity, Equity, and Inclusion Committee through a competitive process. Plus, assistants to the committee have been selected to support key areas of activity. All new volunteers to the energetic team are very welcome.

\section{References}

Berhe, A.A., Barnes, R.T., Hastings, M.G., Mattheis, A., Schneider, B., Williams, B.M. and Marín-Spiotta, E., 2022. Scientists from historically excluded groups face a hostile obstacle course. Nature Geoscience, 15(1), pp.2-4.

https://www.nature.com/articles/s41561-021-00868-0

Bernard R.E., and Cooperdock E.H.G., 2018. No progress on diversity in 40 years. Nature Geoscience, 11, pp. 292-295. https://doi.org/10.1038/s41561-018-0116-6

Bhopal, K. and Pitkin, C., 2020. 'Same old story, just a different policy': race and policy making in higher education in the UK. Race Ethnicity and Education, 23(4), pp. 530-547. https://doi.org/10.1080/13613324.2020.1718082

EAG DEI Committee, 2021a. Professional Culture: Let's Talk Tackling of Inequity, Injustice, and Absent Talent. EAG Blog. https://tinyurl.com/4e8e3vu8

EAG DEI Committee, 2021b. Strengthening Geochemistry through Community Action and Wider Influence. Elements, 17(4), pp. 282-283.

http://www.elementsmagazine.org/archives/e17 4/e17 4 soc EAG.pdf

Geochemical Society, 2021. Nominate a Colleague for a Geochemical Society Award or Special Lecture. Elements, 17(3), pp. 206-207.

http://www.elementsmagazine.org/archives/e17 $3 /$ e17 3 soc GS.pdf

Haacker, R., Burt, M. and Vara M., 2022. Moving beyond the business case for diversity, Eos, 103, https://doi.org/10.1029/2022EO220080. 
Henderson, H. and Bhopal, K., 2021. Narratives of academic staff involvement in Athena SWAN and race equality charter marks in UK higher education institutions. Journal of Education Policy. https://doi.org/10.1080/02680939.2021.1891576

Limas, J.C., Corcoran, L.C., Baker, A.N., Cartaya, A.E. and Ayres, Z.J., 2022. The Impact of Research Culture on Mental Health \& Diversity in STEM. Chemistry-A European Journal, p.e202102957. https://doi.org/10.1002/chem.202102957

Pourret, O., Anand, P., Arndt, S., Bots, P., Dosseto, A., Li, Z., Carbonne, J.M., Middleton, J., Ngwenya, B. and Riches, A.J., 2021. Diversity, equity, and inclusion: Tackling underrepresentation and recognition of talents in geochemistry and cosmochemistry. Geochimica et Cosmochimica Acta, 310, pp. 363-371. https://doi.org/10.1016/j.gca.2021.05.054

Riches, A.J.V., Pourret, O., and Little, S.H., 2021. Uniting to Advance Diversity, Equity, and Inclusion in a Pandemic and Post-Pandemic World. EAG Blog. https://tinyurl.com/ms5bz2vt

Thomson, R.M., 2022. Advancing equity, diversity, and inclusion: a how-to guide. Physics Today, 75(1), pp. 42-49. https://physicstoday.scitation.org/doi/10.1063/PT.3.4921

Tzanakou, C. and Pearce, R., 2019. Moderate feminism within or against the neoliberal university? The example of Athena SWAN. Gender, Work \& Organization, 26(8), pp. 1191-1211. https://doi.org/10.1111/gwao.12336

Vance, D. 2021. EAG needs you to nominate a colleague for an award today! Message from the EAG President. EAG Blog. https://tinyurl.com/3u2t9ns7

Xiao, Y., Pinkney, E., Au, T.K.F. and Yip, P.S.F., 2020. Athena Swan and gender diversity: a UK-based retrospective cohort study. British Medical Journal open, 10(2), p.e032915. https://bmjopen.bmj.com/content/bmjopen/10/2/e032915.full.pdf 